\title{
Percolation in Cluster-Cluster Aggregation Processes
}

\author{
Anwar Hasmy ${ }^{1,2}$ and Rémi Jullien ${ }^{1}$ \\ ${ }^{1}$ Laboratoire de Science des Matériaux Vitreux, UA 1119 CNRS, Université Montpellier II, Place \\ Eugène Bataillon, 34095 Montpellier Cedex 5, France \\ ${ }^{2}$ Laboratoire de Physique des Solides, Université Paris-Sud, Centre d'Orsay, 91405 Orsay, France
}

(September 11, 2018)

\begin{abstract}
Numerical simulations of Diffusion-Limited and Reaction-Limited ClusterCluster Aggregation processes of identical particles are performed in a twodimensional box. It is shown that, for concentrations larger than a characteristic gel concentration, the morphology of the resulting spanning cluster at the gel time $t_{g}$ exhibits a crossover length $L_{c}$ between percolation $\left(l>L_{c}\right)$ and aggregation $\left(l<L_{c}\right) . L_{c}$ vanishes when increasing $c$, and, at a critical concentration value $c_{p}$ (where $L_{c} \rightarrow 0$ ) the entire spanning cluster scales as the percolating cluster obtained by standard percolation. Even if for $c>c_{p}$ the long-range correlations are similar to that of percolation, the vanishing links in the structure suggest that an homogeneous regime appears at small scales.
\end{abstract}

PACS numbers: 61.43.H, 64.60.A

Typeset using REVTEX 


\section{INTRODUCTION}

The aggregation processes of small particles have attracted a great theoretical interest in the last decade [1 4], due to their wide range of applications. On can distinguish ParticleCluster aggregation, whose prototype is the Witten-Sander model [5], and Cluster-Cluster aggregation [6, [7. While the former model applies to field-induced growth processes, such as electrodeposition [8,9], dielectric breakdown [10], viscous fingering [11,12], etc..., the latter explains true aggregation processes such as polymerisation 13, 14, oil in water emulsions [15], soot particles in flames [16], flocculation of colloidal particles [17 21]. Among Cluster-Cluster aggregation processes one distinguishes diffusion-limited cluster-cluster aggregation (DLCA) [6:7] and chemically-limited (also called reaction-limited) aggregation (RLCA) [22 24] which correspond respectively to fast (fully screened) and slow (partially screened) aggregation of colloids [17].

The CCA processes leads to a flocculation regime when the concentration is smaller than a characteristic gel concentration $c_{g}$, and to a gelation regime otherwise. In flocculation regime it remains a single fractal cluster at the end of the aggregation process with a fractal dimension $D$ equal to 1.45 in DLCA case (and 1.65 in RLCA case). In the gelation regime it appears an "infinite" cluster at a given time $t_{g}$ (hereafter refered as the gelling cluster). When working with a finite (but large) box, such a cluster is usually defined as touching the box from edge to edge as in the percolation theory [25]. Since both physical problems are quite similar, it is tempting to explain the infinite cluster formation in DLCA by the percolation theory which excludes all dynamical phenomena and this has been done by several authors [26 31]. The fractal dimension of the infinite cluster of DLCA has been found to be different than the one of percolation [26,27] and it has been argued that this discrepancy is due to dynamical effects. However, recent aggregation experiments [32] on attractive coagulated particles suggest that percolation transition occurs at the particle concentration 0.42. Therefore the question is still open: are CCA models and percolation theory compatible? 
In this paper we show that the infinite cluster obtained at $t_{g}$ in CCA models exhibits a crossover length $L_{c}$ between percolation and aggregation, for concentration values $c$ larger than the gel concentration $c_{g}$. Furthermore, we show that $L_{c}$ vanishes when increasing $c$, and for $c \approx 0.5$ the percolation regime shows up at all length scales in the infinite cluster. These results suggest that CCA processes can be viewed as an irreversible percolation phenomenom, as the invasion percolation model without trapping [33, 34] and the model for a diffusion front [35.

\section{THE MODEL}

The two-dimensional Diffusion-limited Cluster-Cluster Aggregation model consists in a Monte Carlo algorithm which builds clusters on a lattice within a square box of edge length $L$. Initially particles are distributed randomly (but uniformily in the space) on the lattice sites up to volume fraction (concentration) $c$. In order to insure that the diffusion coefficient of the clusters varies as the inverse of their radius $R$, a particle (or a cluster) is chosen randomly according to a probability:

$$
p_{n_{i}}=\frac{n_{i}^{\alpha}}{\sum_{i} n_{i}^{\alpha}}
$$

where $\alpha(=-1 / D)$ is the kinetical exponent. Then the cluster performs a translational motion by one unit (taking account Periodic Boundary Conditions, PBC) in any of the four directions $\pm 1, \pm 1$ chosen at random. If the cluster does not collide with another, the displacement is performed and the algorithm goes on by choosing again another cluster. If a collision occurs between two clusters they stick together forming a new large cluster. In our simulation we have considered that collision occurs when a particle of a cluster tries to occupy a particle of another cluster. In that case the cluster is not displaced but a bond is established between the two contacting particles. If there are more than one collision at a given motion, only one bond is chosen at random. This trick has used by Kolb in a reversible

diffusion-limited cluster aggregation model [28]. This important variant implies that there 
are no loops (as in off-lattice DLCA [36]) and that there is no intrinsic percolation at the begining of the process: the intitial concentration can be varied up to unity.

For concentrations larger than $c_{g}$ there exists a gel time $t_{g}$ where a cluster becomes infinite. This gelling cluster is stored for a numerical analysis, and to compare, we leave the aggregation process continuing up to the time where it remains only one cluster (hereafter refered as the final cluster). In the case of Reaction Limited Cluster-cluster Aggregation [22 24], in addition to the algorithm described above, it is introduced a sticking probabilty $p(\ll 1)$. after a collision a new bond is created only if a random number (uniformly distributed between zero and one) is smaller than $p$. Here, we have performed two-dimensional simulations in boxes of different sizes up to $L=240$.

\section{RESULTS AND DISCUSSION}

Typical results that compare qualitatevely the resulting morphologies of the gelling cluster $(\mathrm{G})$ and the final cluster $(\mathrm{F})$ in DLCA are are illustrated in figure 1 . In the case of the gelling cluster the other remaining clusters have been discarded and therefore are not shown. Note that the morphology of the cluster shown in figure 1a is strongly reminiscent of that of a percolating cluster as obtained in standard percolation theory [25] when occupying randomly the sites of a square lattice with the percolation probability $p_{c}=0.59273$. To make quantitative comparaisons between $\mathrm{G}$ and $\mathrm{F}$, we have calculated the mass $M$ dependent box size $l$ using the mass-counting algorithm [37]. In figure 2 we show the $\log$-log plot of $M$ versus $l$ for the same concentration than those considered in figure 1. The resulting slope indicates that $\mathrm{M}$ scales as $l^{D_{p}}$ and $l^{d}$, for $\mathrm{G}$ (open circles) and $\mathrm{F}$ (black squares), respectively. $D_{p}(=1.89)$ is close to the fractal dimension of the percolating cluster in two dimensions, and $d(=2)$ is the spatial dimension.

If the mass of the gelling cluster $\mathrm{G}$ or the mass of the final cluster $\mathrm{F}$ scales as $l^{D}$ we

might be tempted to conclude that the quantity $m=\frac{M(l)}{c l^{D}}$ should not depend on $l$. To test this we show, in figure 3, the curves giving $m$ as a function of $l$. Figures $3 \mathrm{a}$ and $3 \mathrm{c}$ 
corresponds to $\mathrm{G}$ for $D=D_{p}$ and $\mathrm{D}=\mathrm{d}$, respectively and figures $3 \mathrm{~b}$ and $3 \mathrm{~d}$ correspond to $\mathrm{F}$ for $D=D_{p}$ and $\mathrm{D}=\mathrm{d}$, respectively. One observes on these figures that $m$ is independent on $l$ only in cases $3 \mathrm{a}$ and $3 \mathrm{~d}$ and for sufficiently large concentrations, confirming our first guess of different fractal dimensions, 1.89 and 2.00, for $\mathrm{G}$ and $\mathrm{F}$ respectively. Figures $3 \mathrm{~b}$ and 3c are shown here as conter-examples to illustrate the high precisions on these estimates for the fractal dimensions. In general, i.e. for not too large concentrations, one can define a $c$-dependent crossover length $L_{c}$ such that $m$ becomes independent on $l$ only for $l>L_{c}$. For $l<L_{c}$, another linear regime is observed, which is better extended at very low concentration, with a slope of about -0.4 and -0.5 in cases $3 \mathrm{a}$ and $3 \mathrm{~d}$, respectively, corresponding to the fractal dimension $D \simeq 1.89-0.4 \simeq 2.00-0.5 \simeq 1.5$, close to the one of DLCA clusters. Therefore $L_{c}$ defines a change of scaling regime between DLCA for short lengths and either percolation $(\mathrm{G})$ or homogeneity $(\mathrm{F})$ for large lengths. In fact, $L_{c}$ should be proportional to the characteristic length correlation $\xi$ (or average size) of DLCA fractal aggregates [36]. Note that in all cases $L_{c}$ vanishes for $c \approx 0.5$, and this is the reason of the absence of crossover in the curves reported in figure 2.

When estimating the fractal dimension of the aggregates $D_{a g g}$ in the range of lengths corresponding to the aggregation regime $\left(l<L_{c}\right)$, we found, in both cases $\mathrm{G}$ and $\mathrm{F}$, that it increases significantly with concentration (the data are reported in figure 4). Those results confirm previous conclusions in two dimensions [38] as well as in three dimensions [39]. As a consequence the $c$-dependence of $L_{c}$ cannot be annalysed as a simple scaling relation. Moreover, the discrete values of $l$ that require mass-counting calculations, and finite size effects, impedes us to determine $L_{c}$ within a sufficiently small range of error. Anyway, all the results depicted in figs. 2 and 3 suggest that, at least for $l>L_{c}$ the mass of the gelling cluster $\mathrm{G}$ scales with $l$ as a standard percolation cluster. However such results are not sufficient to insure that their morphologies are the same. It is well known that there exist additional quantities (different and independent on the fractal dimension) to characterize a percolating cluster. In principle, the morphology is entirely characterized by an infinite set of exponents [25,40], but here we shall focuse on two particular exponents: the fractal 
dimensions of the "backbone" $D_{b b}$ 41] and the fractal dimension of the "links" 42]. The backbone of a cluster is the ensemble which remains after removing dead-ends (or danglingends). For a percolating cluster in two dimension the fractal dimension of the backbone $D_{b b}$ is 1.61. The links (also called red bonds) are the sites of the cluster that are singly connected, that is, if we take out a link the connection between the entire cluster is broken. For a percolating cluster in two dimensions the fractal dimension of links $D_{l}$ is equal to 0.75 .

In order to identify the backbone and the links of a given cluster, we have been obliged (due to the PBC considered in the CCA simulations) to span the cluster out of the box, taking account of PBC. Then, we have applied to the new cluster configuration (with size larger than the box size $L$, in almost all situations) a procedure suggested by Hermann et al. 433 to identify the backbone and the links. Finally, the resulting backbone (and links) is unspanned and returned inside the original box. In figures 1, the sites depicted with strong grey and black colors denotes the backbone and the links, repectively.

We have calculated the fractal dimension of the backbone $D_{b b}$ for both $\mathrm{G}$ and $\mathrm{F}$ clusters and different concentration values. The results are depicted in figure 5 for two box sizes length $L=90$ (black symbols) and 120 (open symbols). Note that for small concentration values the $D_{b b}$ values, for both $\mathrm{G}$ and $\mathrm{F}$ clusters, are quite similar. This can be understood, since, as mentioned above, the fractal dimension in the aggregation regime is almost the same in both cases. This conclusion is more convincing for $L=120$, but for larger $c$ values, $D_{b b}$ of the gelling cluster $\mathrm{G}$ becomes smaller than $D_{b b}$ of the final cluster F. Furthermore, in the $\mathrm{G}$ case, $D_{b b}$ reaches the value 1.61 (the same value than for a percolating cluster) for $c \approx 0.5$. For $c>0.5, D_{b b}$ saturates to 1.89 (the value of the fractal dimension of a percolating cluster). In the $\mathrm{F}$ case, the same kind of saturation phenomenon occurs but, for $c>0.5, D_{b b}$ saturates to the spatial dimension $d$. The increasing value of $D_{b b}$ suggests that the backbone structure is mainly reflecting the fractal aggregate structure (Which also increases with concentration as shown in figure 4). It is only when the crossover length $L_{c}$ vanishes that the backbone structure becomes characteristic of the one of a percolating cluster, since $D_{b b}$ varies from 1.61 to 1.89 as shown in figure 5 . 
In figure 6 it is shown that for $c$ smaller than about 0.5 , the fractal dimension $D_{l}$ of the links is approximately equal to 1 , suggesting that the mass of the links scales with $l$ in a trivial manner. However, for $c \approx 0.5, D_{l}$ reaches the value 0.75 as in a percolating cluster. The $D_{l}$ undependence on the box size $L$ suggests that there exist a threshold concentration, close to 0.5 where the entire $\mathrm{G}$ cluster scales exactly as a percolating cluster. When $c$ is increased above this threshold, $D_{l}$ vanishes, similarly to the percolation theory when the occupation probability $p$ is increased above $p_{c}$ 40]. The vanishing links in the gelling cluster for large $c$ values suggest that, at very small scales, the system becomes homogeneous, even if, at large distances, the system scales as a percolating cluster as shown in figure 3 .

In order to appreciate the degree of generality of our results, we have also performed some calculations in the reaction-limited case (RLCA). In figure 7 we have reported the $m(l)$ curves and it can be shown that they exhibit the same qualitative behavior than in the DLCA case (figure 3). These results suggest that the percolation scaling could exist in other kinds of CCA processes such as the ballistic-limited [44], the convection-limited 455] and the fluctuating bond 46] aggregation models.

\section{CONCLUSION}

In this paper we have shown that the infinite cluster obtained at the gel time $t_{g}$ in CCA models exhibits a crossover length $L_{c}$ between aggregation and percolation. Moreover, $L_{c}$ vanishes at a critical concentration $c_{p}(\simeq 0.5)$ where the mass of the entire system (and its backbone and links) scales as for a percolating cluster obtained at $p_{c}$. For $c>c_{p}$ the percolation regime persists at least at large scales, because the vanishing links suggest that an homogeneous regime apperas at small scales. The value obtained here for $c_{p}$ is close to the value reported in the above mentioned experimental work $[32](\simeq 0.42)$, but typical statistical fluctuations to determine a critical value impedes us to insure if there are some relation between these two critical probabilities. It might also be worth finding a relation between $c_{p}$ and the critical probability of the site percolation threshold $\left(p_{c}=0.59273\right)$ on 
a square lattice. On the other hand, the fact that close to $c_{p}$ the fractal dimension of the links $D_{l}$ becomes equal to that of the percolating clusters suggests that the critical behavior in both cases are the same, since the correlation lenght exponent $\nu$ is given by $\frac{1}{D_{l}} 47$. Preliminary calculations on a cubic lattice suggest that the results reported here are quite general and extend in three dimensions.

One of us (A. H.) would like to acknowledge support from CONICIT (Venezuela). The numerical calculations where done on the computers of the CNUSC (Centre Universitaire Sud de Calcul), Montpellier, France, with support from CNRS. 


\section{REFERENCES}

[1] F. Family and D. P. Landau, "Kinetics of Aggregation and Gelation", North-Holland, Amsterdam (1984).

[2] R. Jullien and R. Botet, "Aggregation and Fractal Aggregates", World Scientific (Singapore, 1987).

[3] Phase Trans. and Crit. Phenom., 12, 335 (1988).

[4] T. Vicsek, "Fractal Growth Phenomena", World Scientific (Singapore, 1989).

[5] T. A. Witten and L. M. Sander, Phys. Rev. Lett., 47, 1400 (1981).

[6] P. Meakin, Phys. Rev. Lett., 51, 1119 (1983).

[7] M. Kolb, R. Botet and R. Jullien, Phys. Rev. Lett., 51, 1123 (1983).

[8] M. Matsushita, M. Sano, Y. Hayakawa, H. Honjo and Y. Sawada, Phys. Rev. Lett., 53, $286(1984)$.

[9] J. Mach, F. Mas and F. Sagués, Europhys. Lett., 25, 271 (1994).

[10] L. Niemeyer, L. Pietronero and H. J. Wiesmann, Phys. Rev. Lett., 52, 1033 (1984).

[11] A. Arnéodo, Y. Couder, G. Grasseau, V. Hakim and M. Rabaud, Phys. Rev. Lett., 63, 984 (1989).

[12] H. La Roche, J. F. Fernández, M. Octavio, A. G. Loeger and C. J. Lobb, Phys. Rev. A, 44, 6185 (1991).

[13] B. Cabane, M. Dubois and R. Duplessix, J. Physique, 48, 2131 (1987).

[14] J. C. Pouxviel, J. P. Boilot, A. Dauger and A. Wright, J. of J. of Non-Cryst. Solids, 103, 331 (1988).

[15] J. Bibette, T. G. Mason, H. Gang and D. A. Weitz, Phys. Rev. Lett., 69, 981 (1992). 
[16] J. Cai, N. Lu and C. M. Sorensen, Langmuir, 9, 2861 (1993).

[17] D. A. Weitz, J. S. Huang, M. Y. Lin and J. Sung, Phys. Rev. Lett. 53, 1657 (1984).

[18] M. L. Broide and R. J. Cohen, Phys. Rev. Lett., 64, 2026 (1990).

[19] M. Foret, J. Pelous and R. Vacher, J. de Physique I, 2, 791 (1992).

[20] D. J. Robinson and J. C. Earnshaw, Phys. Rev. Lett., 71, 715 (1993).

[21] C. Allain, M. Cloitre and M. Wafra, Phys. Rev. Lett., 74, 1478 (1995).

[22] R. Jullien and M. Kolb, J. of Phys. A, 17. L639 (1984).

[23] M. Kolb and R. Jullien, J. de Phys. Lettres, 45, L977 (1984).

[24] W. Brown and R. C. Ball, J. of Phys. A, 18, L517 (1985).

[25] D. Stauffer, "Introduction to Percolation Theory", Taylor and Francis (London, 1985).

[26] M. Kolb and H. J. Herrmann, J. of Phys. A, 18, L435 (1985).

[27] H. J. Herrmann and M. Kolb, J. of Phys. A, 19, L1027 (1986).

[28] M. Kolb, J. of Phys. A, 19, L263 (1986).

[29] P. Jensen, P. Melinon, A. Hoareau, J. X. Hu, B. Cabaud, M. Treilleux, E. Bernstein and D. Guillot, Physica A, 185, 104 (1992).

[30] T. Nicolai, D. Durand and J. C. Gimel, Phys. Rev. B, 50, 16357 (1994).

[31] J. C. Gimel, D. Durand and T. Nicolai (unpublished).

[32] Q-H Wei, M. Han, C-H Zhou, N-B Ming, Phys. Rev. E, 49, 4167 (1994).

[33] D. Wilkinson and J. Willemsen, J. of Phys. A, 16, 3365 (1983).

[34] R. Paredes and M. Octavio, Phys. Rev. A, 46, 994 (1992).

[35] B. Sapoval, M. Rosso and J. F. Gouyet, J. de Phys. Lettres, 46, L149 (1985). 
[36] A. Hasmy, E. Anglaret, M. Foret, J. Pelous and R. Jullien, PRB, 50, 1305 (1994).

[37] J. Feder, "Fractals", Plenum (New York, 1988).

[38] H. F. van Garderen, W. H. Dokter, T. P. M. Beelen, R. A. van Santen, E. Pantos, M. A. J. Michels and P. A. J. Hilbers, J. Chem. Phys., 102, 480 (1995).

[39] A. Hasmy and R. Jullien, J. of Non-Cryst. Solids (to be published).

[40] M. B. Isichenko, Rev. of Mod. Phys., 64, 961 (1992).

[41] H. J. Herrmann and H. E. Stanley, Phys. Rev. Lett., 53, 1121 (1984).

[42] R. Pike and H. E. Stanley, J. of Phys. A, 14, L169 (1981).

[43] H. J. Herrmann, D. C. Hong and H. E. Stanley, J. of Phys. A, 17, L261 (1984).

[44] R. C. Ball and R. Jullien, J. de Phys. Lettres, 45, L1031 (1984).

[45] P. B. Warren, R. C. Ball and A. Boelle, Europhys. Lett., 29, 339 (1995).

[46] R. Jullien and A. Hasmy, Phys. Rev. Lett. (to be published).

[47] A. Coniglio, J. of Phys. A, 15, 3829 (1982). 


\section{FIGURES}

FIG. 1. Typical configurations for a (a) gelling cluster $\mathrm{G}$ and (b) final cluster $\mathrm{F}$, for $\mathrm{c}=0.5$ and $\mathrm{L}=120$. The sites shown in dark grey and black belong to the backbone and the links, respectively.

FIG. 2. Log-Log plot of $M$ versus $l$ for $c=0.5$ and $L=240$, for the gelling cluster $\mathrm{G}$ (open circles) and the final cluster F (black squares). These curves result from averages over 40 simulations.

FIG. 3. Log-Log plot of $m\left(=M(l) / l^{D}\right)$ versus $l$ for $L=240$ and different concentration values $(0.1,0.2,0.3, \ldots, 0.8$, from top to bottom) in the DLCA case. (a) and (c) correspond to G-cluster for $D=D_{p}$ and $D=d$, respectively. (b) and (d) correspond to F-cluster for $D=D_{p}$ and $D=d$, respectively. These curves result from averages over 40 simulations.

FIG. 4. Fractal dimension of the aggregates $D_{a g g}\left(l<L_{c}\right)$ versus c, for $L=90$ (black squares), $L=120$ (black triangles) and $L=240$ (open diamonds), resulting from averages over 80, 60 and 40 simulations, respectively.

FIG. 5. Fractal dimension of the backbone $D_{b b}$ versus c, for $L=90$ (black symbols) and $L=120$ (open symbols). Square symbols and circle symbols denote $D_{b b}$ for the gelling cluster and the final cluster, respectively. These data results from averages over 80 and 60 simulations, respectively.

FIG. 6. Fractal dimension of the links $D_{l}\left(l<L_{c}\right)$ versus c, for $L=60$ (open circles), $L=90$ (black squares) and $L=120$ (open diamonds), resulting from averages over 120, 80 and 60 simulations, respectively.

FIG. 7. Log-Log plot of $m\left(=M(l) / c l^{D}\right)$ versus $l$ for $L=120$ and different concentration values $(0.2,0.4, \ldots, 1$, from top to bottom) for the RLCA case (with a sticking probability equal to 0.005). (a) and (c) correspond to G-cluster for $D=D_{p}$ and $D=d$, respectively. (b) and (d) correspond to F-cluster for $D=D_{p}$ and $D=d$, respectively. These curves result from averages over 20 simulations. 

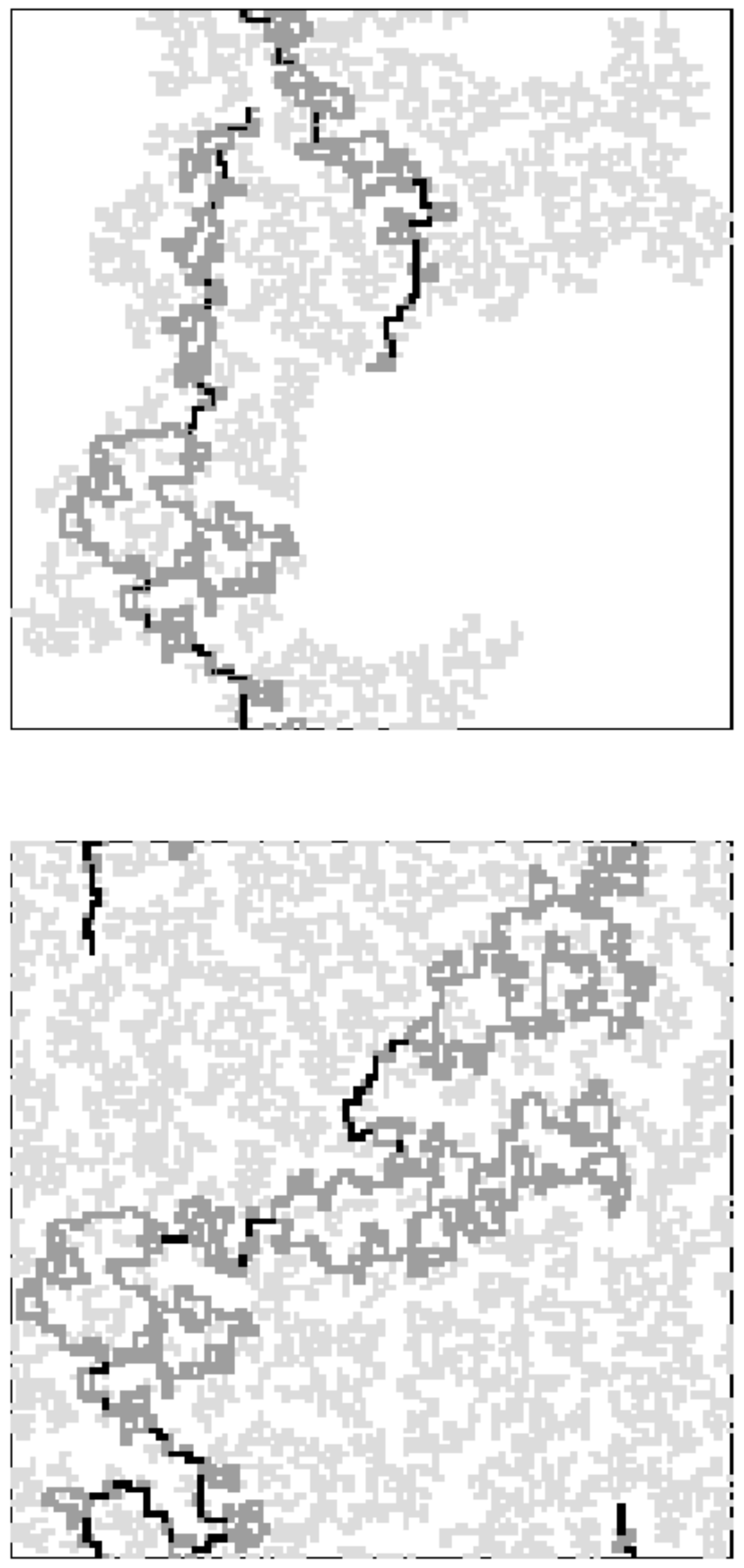
Fig 2

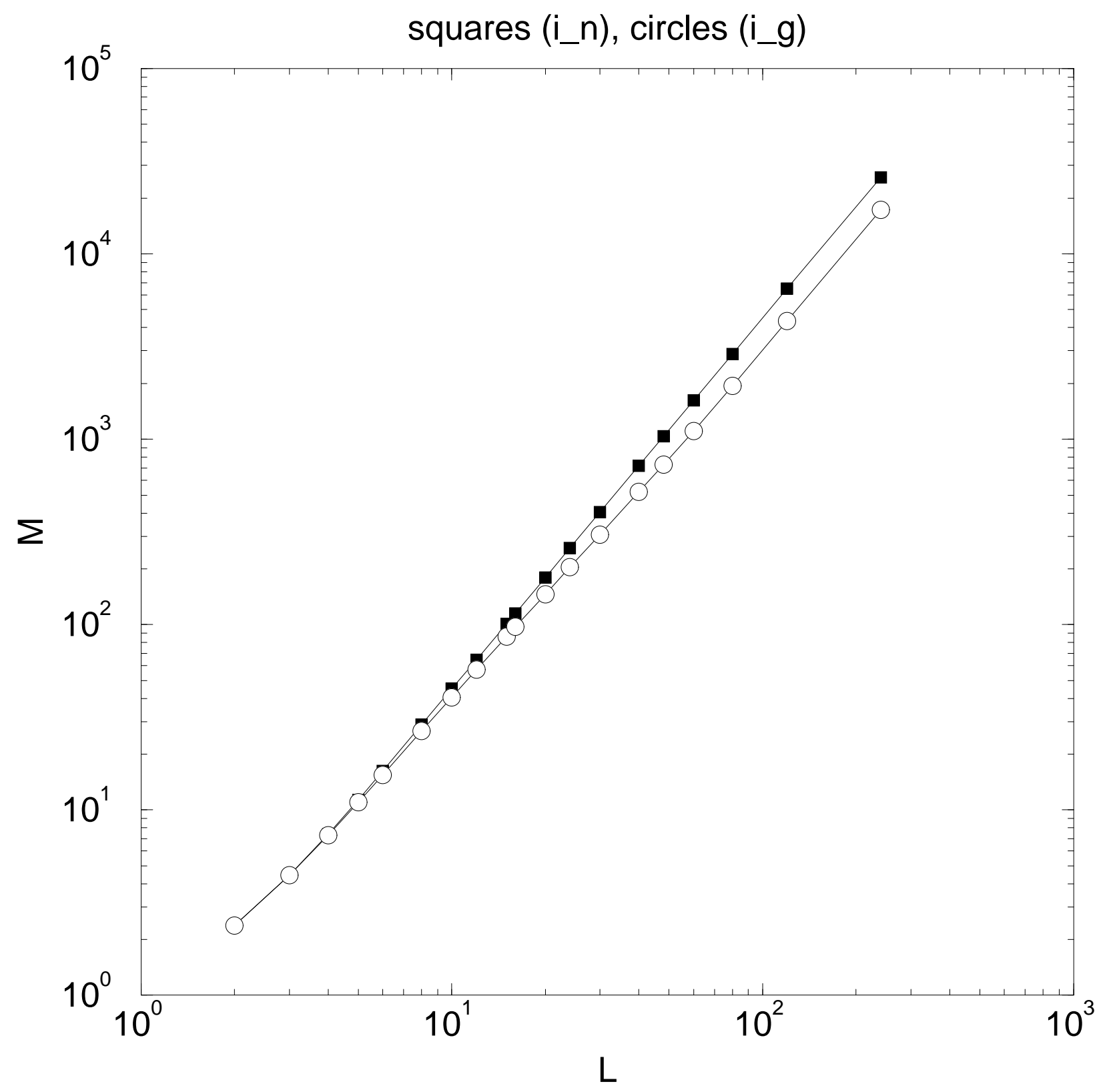


FIG 3

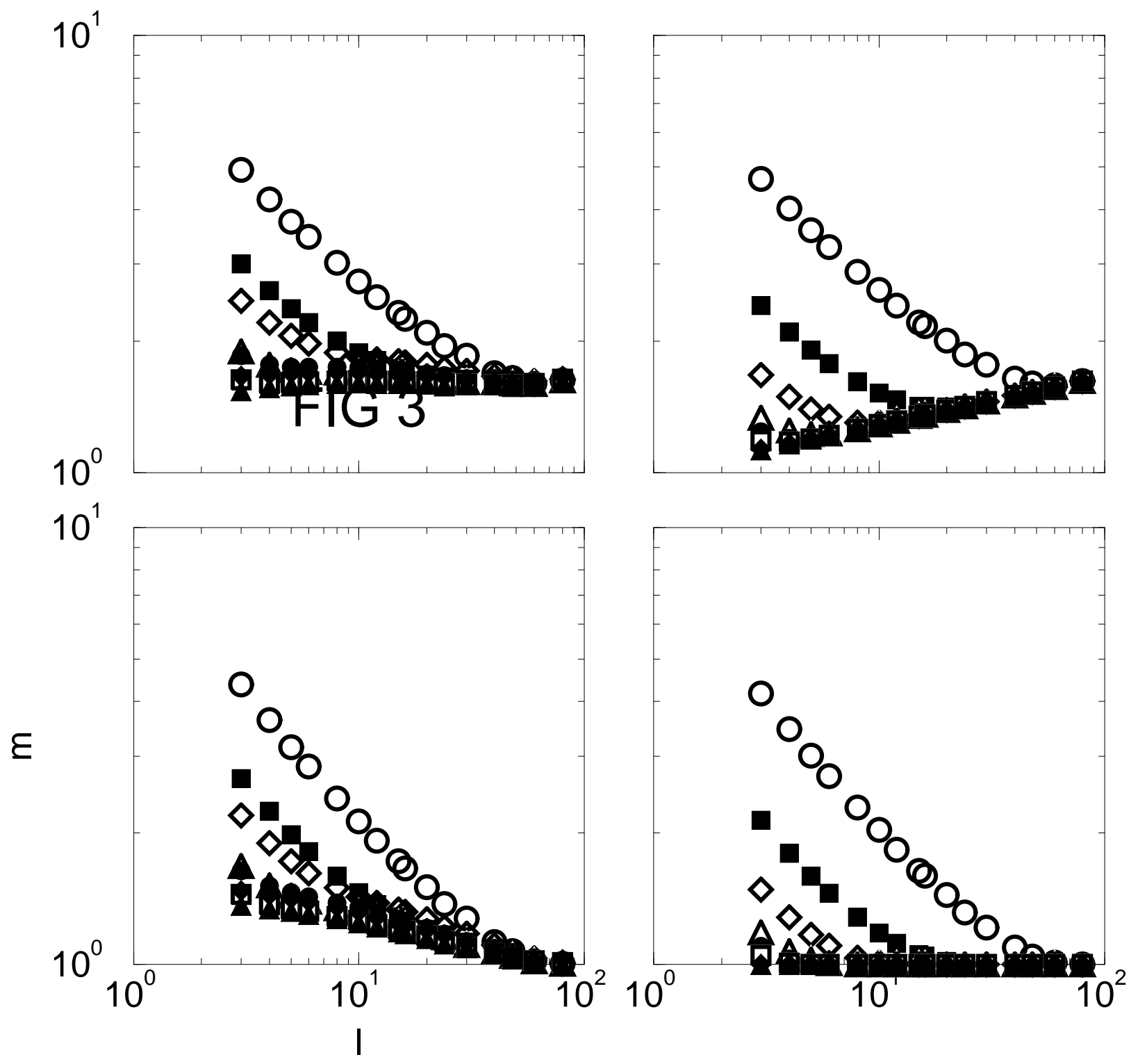


Fig. 4

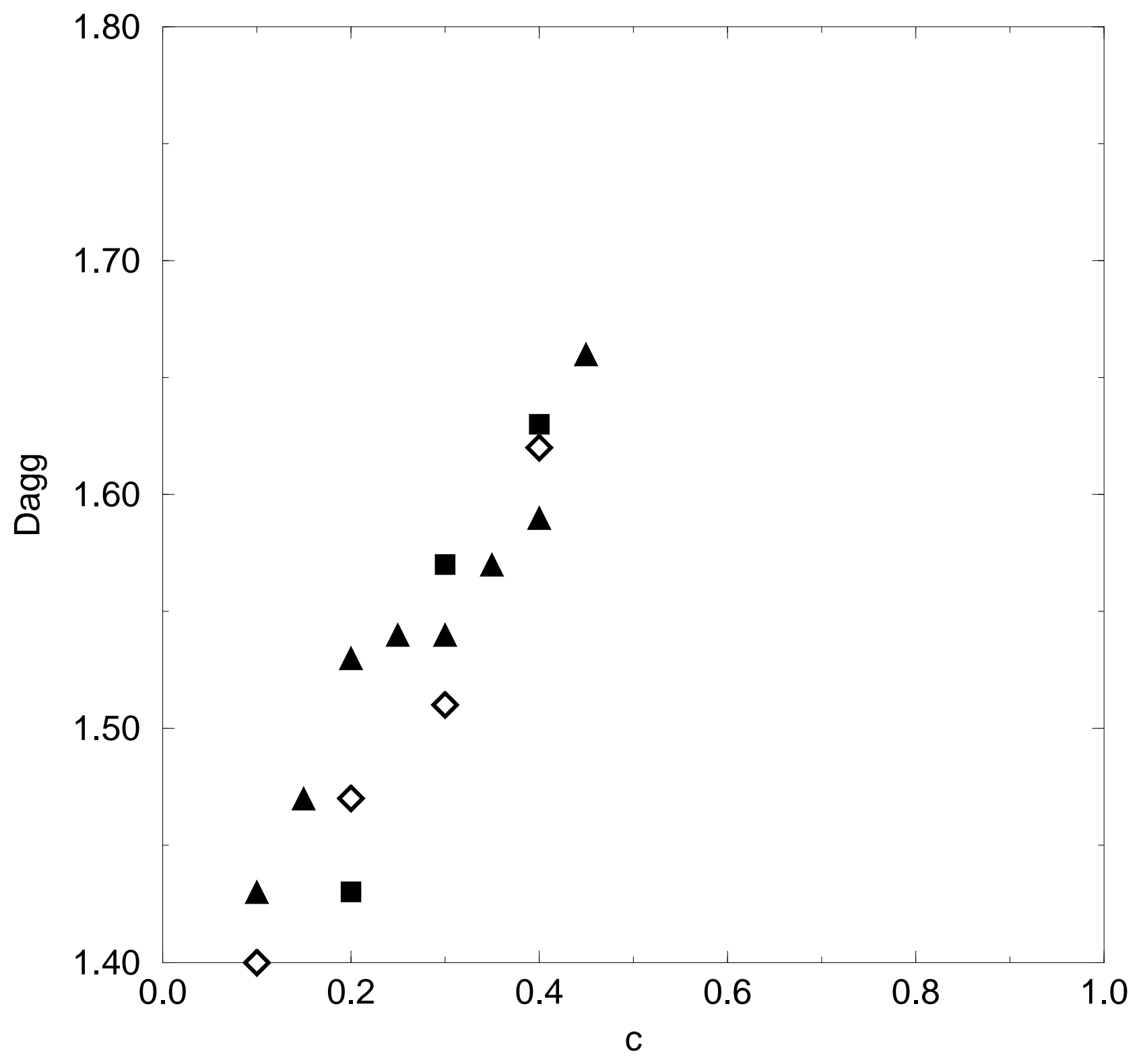


FIG 5

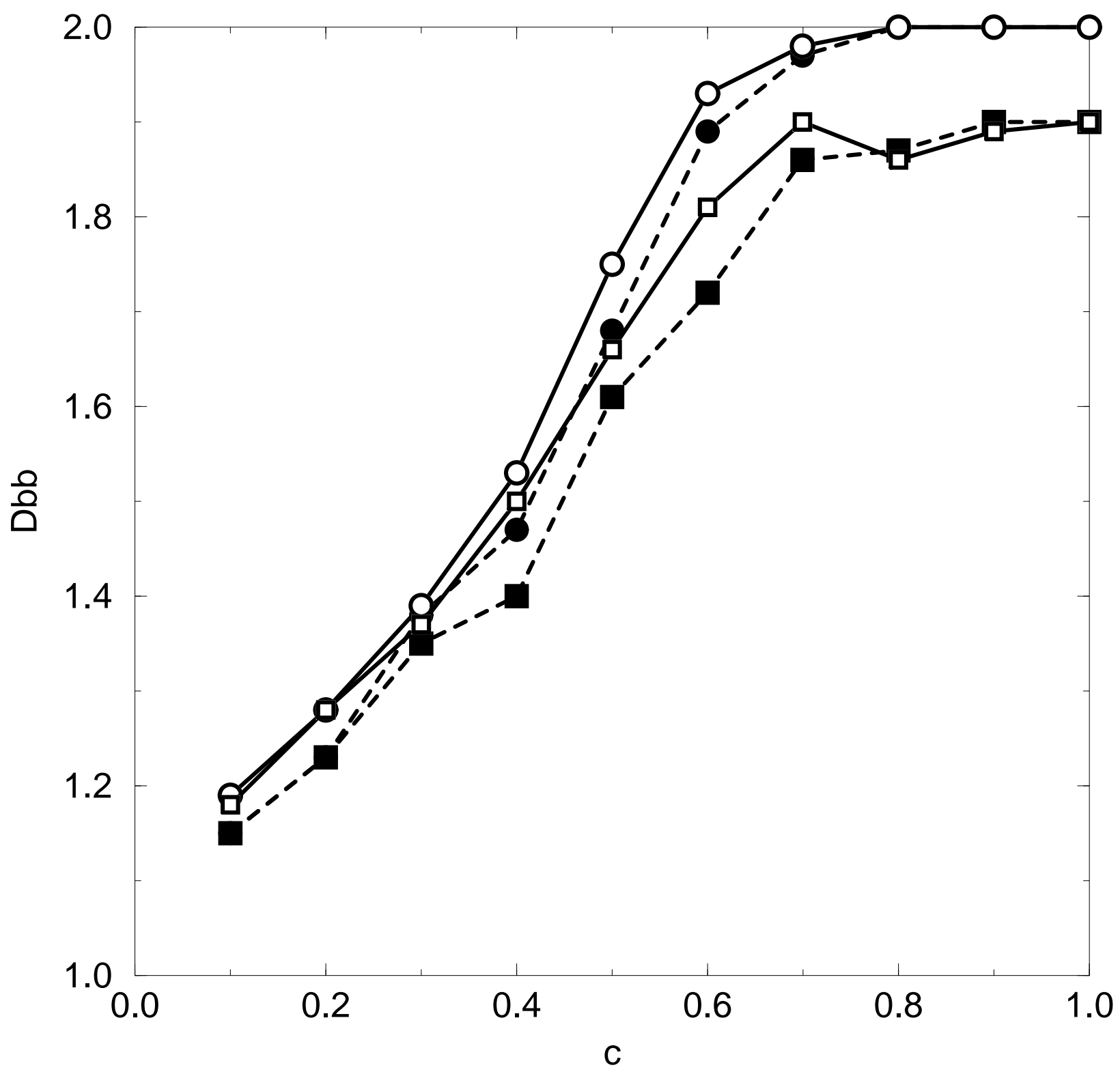


FIG 6

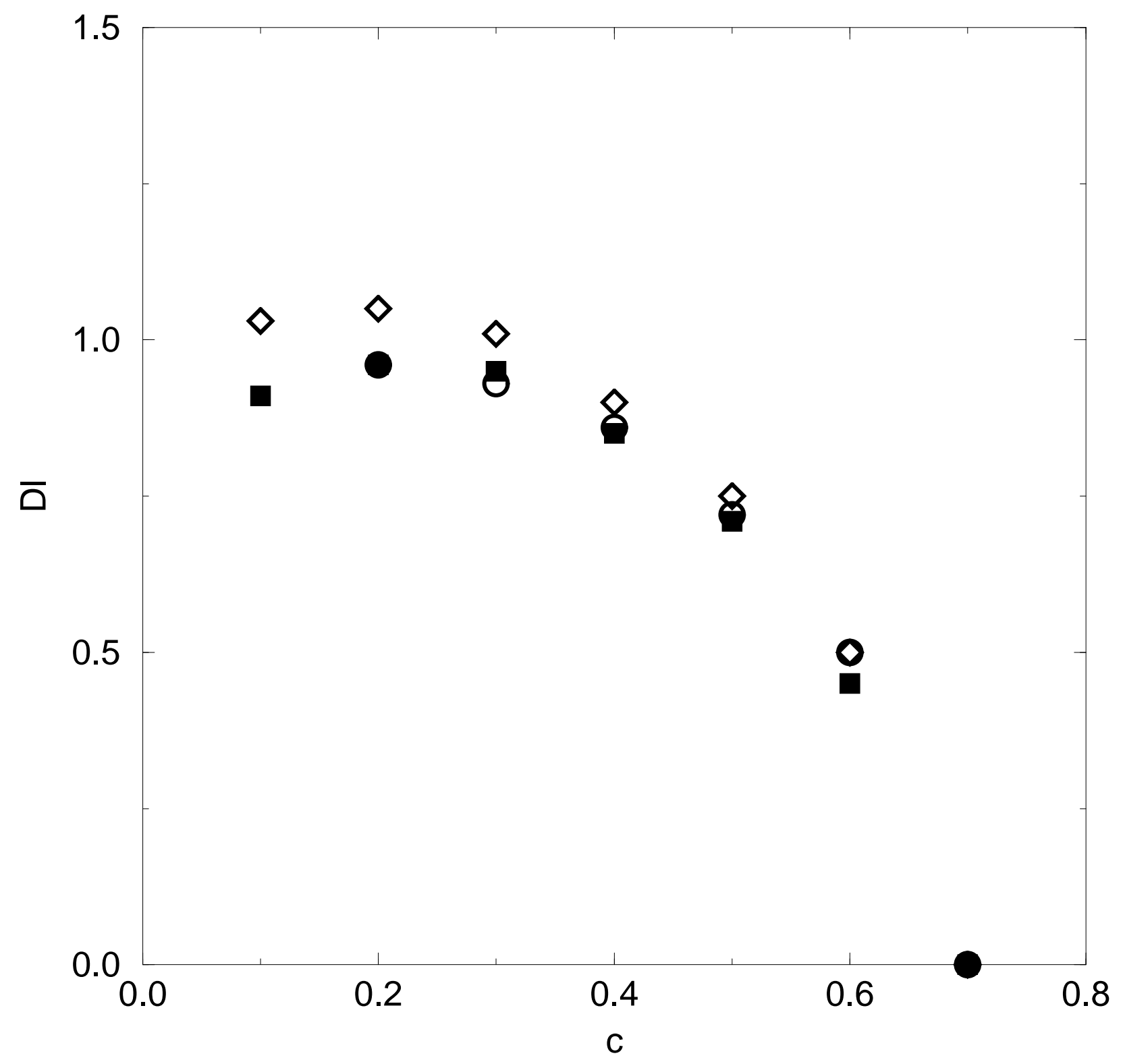


Fig. 7

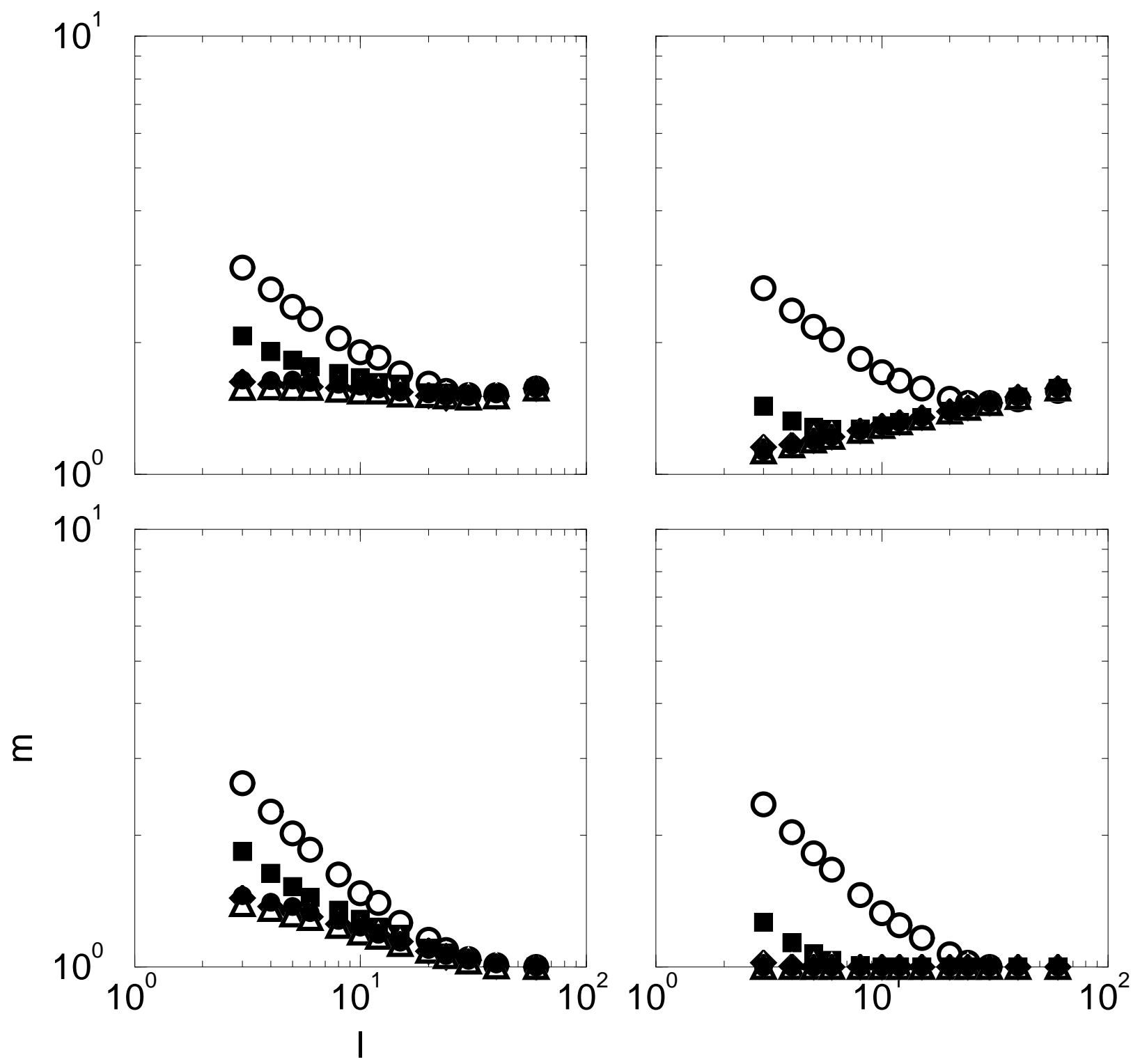

